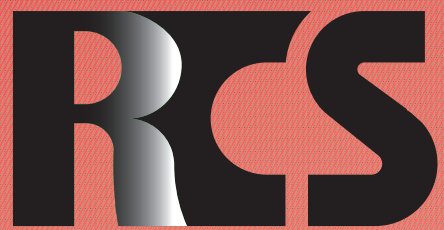

Depósito legal ppi $201502 Z U 4662$

Esta publicación científica en formato digital es continuidad de la revista impresa Depósito Legal: pp $197402 Z U 789$

- ISSN: 1315-9518 • ISSN-E: 2477-9431

Revista de Ciencias Sociales

Universidad del Zulia. Revista de la Facultad de Ciencias Económicas y Sociales Vol. XXVII. No. 2

Abril-Junio 2021

Esta publicación científica en formato digital es continuidad de la revista impresa Depósito Legal: pp $197402 Z$ Z789 ISSN: 1315-9518 


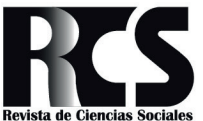

Revista de Ciencias Sociales (RCS). FCES - LUZ

Vol. XXVII, No. 1, 2021, 19-38 Abril-Junio

- ISSN: 1315-9518 • ISSN-E: 2477-9431
Como citar APA: Carrillo-Hidalgo, I., Casado-Montilla, J., y Pulido-Fernández, J. I. (2021). Desarrollo sostenible del oleoturismo: Un análisis de la colaboración entre actores. Revista de Ciencias Sociales (Ve), XXVII(2), 19-38.

\title{
Desarrollo sostenible del oleoturismo: Un análisis de la colaboración entre actores
}

\author{
Carrillo-Hidalgo, Isabel" \\ Casado-Montilla, Jairo ** \\ Pulido-Fernández, Juan Ignacio ${ }^{* * *}$
}

\section{Resumen}

El oleoturismo surge como una forma de diversificación económica en las regiones productoras de aceite de oliva, muy dependientes de la agricultura. Para lograr un desarrollo sostenible es fundamental la colaboración entre los actores que influyen y se ven afectados por esta actividad, puesto que el turismo debe analizarse como un sistema integrado y estas relaciones favorecen a la industria y al propio destino. Este hecho suscita la necesidad de conocer en profundidad cual es la predisposición que presentan las empresas oleoturísticas a colaborar y cooperar entre ellas y con el resto de actores involucrados en la actividad turística, siendo el principal objetivo de este trabajo. Para tal fin, a través de un estudio descriptivo y aplicando una compleja encuesta, se entrevistaron gerentes de empresas situadas en Andalucía-España, pioneras en la diversificación de su actividad hacia el oleoturismo. Los resultados muestran, que la prioridad de colaborar de las empresas es baja, siendo el sector público el actor preferido y la predisposición a invertir capital económico en redes de colaboración es mínima. Se concluye que, en materia de colaboración entre las partes interesadas del mercado oleoturístico, las empresas con mejor posicionamiento en esta actividad necesitan información, formación e incentivos para desarrollarlo.

Palabras clave: Oleoturismo; sostenibilidad; desarrollo turístico; aceite de oliva; colaboración entre actores.

* Profesora Ayudante Doctor en el Departamento de Economía Financiera y Contabilidad y miembro del Laboratorio de Análisis e Innovación Turística (LAInnTUR) en la Universidad de Jaén, España. E-mail: ihidalgo@ujaen.es (iD ORCID: https://orcid.org/0000-0002-0914-5084

** Estudiante de Doctorado y colaborador en el Laboratorio de Análisis e Innovación Turística (LAInnTUR) en la Universidad de Jaén, España. E-mail: jcasado@ujaen.es (iD) ORCID: https://orcid. org/0000-0003-1250-1534

**** Profesor Titular en el Departamento de Economía y Director del Laboratorio de Análisis e Innovación Turística (LAInnTUR) en la Universidad de Jaén, España. E-mail: jipulido@ujaen.es iD ORCID: https://orcid.org/0000-0002-9019-726X

Recibido: 2020-12-05 • Aceptado: 2021-02-22 


\title{
Sustainable development of oleotourism: An analysis of the collaboration between actors
}

\begin{abstract}
Oleotourism arises as a form of economic diversification in olive oil producing regions, which are highly dependent on agriculture. To achieve sustainable development, collaboration between the actors that influence and are affected by this activity is essential, since tourism must be analyzed as an integrated system and these relationships favor the industry and the destination itself. This fact raises the need to know in depth what is the predisposition that oil tourism companies present to collaborate and cooperate with each other and with the rest of the actors involved in the tourist activity, being the main objective of this work. To this end, through a descriptive study and applying a complex survey, managers of companies located in AndalusiaSpain, pioneers in the diversification of their activity towards oleotourism, were interviewed. The results show that the priority of companies to collaborate is low, with the public sector being the preferred actor and the predisposition to invest economic capital in collaboration networks is minimal. It is concluded that, in terms of collaboration between the interested parties of the oleotourism market, the companies with the best positioning in this activity need information, training and incentives to develop it.
\end{abstract}

Keywords: Oleotourism; sustainability; tourism development; olive oil; collaboration between actors.

\section{Introducción}

El aceite de oliva, además de ser un producto alimenticio esencial de la dieta mediterránea (Patrimonio Inmaterial de la Humanidad desde 2010), representa parte de la cultura y del patrimonio del área geográfica que comprende la Europa mediterránea, Oriente Medio y norte de África (LópezGuzmán, et al., 2016); pudiendo identificarse como patrimonio gastronómico y beneficiarse de la importancia adquirida por este en relación con el turismo (Henderson, 2009; Lee y Scott, 2015). Por tanto, el aceite cuenta con una serie de características que influyen en diferentes áreas de la sociedad y del conocimiento, como la cultura, salud, ecología, economía y también el turismo (Ruiz, 2010).

El oleoturismo, es la forma de turismo que surge de la necesidad de conocer la manera en que se produce el aceite de oliva o la obtención de la aceituna en sus distintas modalidades, un mayor conocimiento sobre este aceite y sus propiedades, así como realizar y participar en diferentes actividades en las almazaras (Quesada, Molina y Ruiz, 2010).

Se trata de una nueva tipología turística, basada en el conocimiento y disfrute de un tipo de cultivo extensivo en el Mediterráneo, en el que el territorio es uno de los factores clave que determinan la oferta y demanda (Molina, Quesada y Ruiz, 2011), del que España (y la provincia de Jaén en particular) es la máxima productora a nivel mundial $(44,8 \%$ del total) y donde se concentra la mayor superficie mundial destinada a la plantación y explotación del olivar (Consejo Oleícola Internacional [COI], 2018). Además, el oleoturismo, ha sido definido como:

Un viaje de ocio, o de negocios, que requiere necesariamente, al menos, de una pernoctación por parte del viajero, cuyo desplazamiento está motivado por el conjunto de actividades cuya base es el aceite de oliva y la cultura, el patrimonio, el paisaje y las costumbres de la población 
que giran a su alrededor. La combinación de estos componentes tiene el objetivo de cubrir las diversas necesidades de ocio en auge muy específicas, que serán satisfechas, in situ, en el lugar en el que se desarrolle la actividad demandada, variando, en función de ésta, el nivel de involucración necesario por parte del turista para la producciónconsumo de esta tipología turística. (Pulido-Fernández, Casado-Montilla y Carrillo-Hidalgo, 2019, p.5-6)

Siguiendo esta definición, y según la literatura científica analizada, el oleoturismo puede enmarcarse dentro del turismo rural (Duarte, 2010; Duarte y Northcote, 2010; Millán, Morales y Pérez-Naranjo, 2010; Millán, Morales-Fernández y Pérez, 2012; Cañero, et al., 2015); del turismo cultural (Ruiz, 2010), comunitario (Ordoñez y Ochoa, 2020; Loor, Plaza y Medina, 2021); gastronómico (De Salvo, et al., 2013; Orgaz-Agüera, et al., 2018); o del agroturismo (Kizos y Vakoufaris, 2011; Campón-Cerro, et al., 2014; Cañero, et al., 2015). Asimismo, cada vez está más identificado con el turismo industrial en la mayoría de los países europeos precursores de éste, sobre todo Francia y Holanda (Quesada, et al., 2010).

La base del oleoturismo es el olivar, y este, además de los olivos, tiene asociados una serie de recursos como el suelo, el agua, otras plantas y los animales que habitan en los mismos (Alonso y Guzmán, 1999). El medio rural, con los paisajes, el clima y las características propias que integra, forma parte de la experiencia oleoturística, implicando un estilo de vida fruto de una determinada cultura, que contribuye a crear una imagen turística (Aybar, 2004).

El oleoturismo, permite diversificar los ingresos de la población de los destinos, dependientes en su mayoría de la agricultura y puede revitalizar la economía de determinadas áreas rurales, debido a que el turismo es una actividad con capacidad para generar empleo y riqueza, aprovechando los recursos disponibles sin necesidad de saturar el territorio. Para lograr este objetivo, es necesario coordinar y planificar todas las iniciativas relacionadas con el turismo, hacer frente a las amenazas y debilidades que se presentan así como promover la sostenibilidad, ofreciendo un producto diferenciado y competitivo que satisfaga las necesidades de la demanda (Millán, Agudo y Agudo, 2010).

El origen del oleoturismo como explotación turística, surge como una modalidad de turismo inspirada en la del enoturismo y como complemento del turismo genérico (Ruiz, Molina y Martín, 2011), aunque es considerado como una modalidad de turismo rural desarrollada en territorios de explotación olivarera de la cuenca mediterránea, en los que se desarrollan actividades turísticas organizadas en torno al aceite de oliva y entre las que se encuentran: Visitas a campos de cultivo, a almazaras, catas, así como al estudio de la cultura y la historia del aceite (Millán, Morales y Agudo, 2010).

El desarrollo del oleoturismo, permite fortalecer la imagen del aceite de oliva y, al mismo tiempo agregar valor a la cultura de este aceite (Duarte y Northcote, 2010), a través de un mayor conocimiento del mundo que rodea a su extracción y por venta directa de este producto (Duarte, 2010). Sin embargo, la literatura científica (Murgado, 2013; Millán, Arjona y Amador, 2014; Cañero, et al., 2015) ha identificado diferentes problemas y limitaciones a los que se enfrenta el oleoturismo, que siendo un tipo de turismo con un gran potencial (Tregua, D'Auria y MaranoMarcolini, 2018), no logra un desarrollo sostenible pleno (Cañero, et al., 2015), lo que exige estudiar en profundidad diferentes aspectos de las dimensiones económica, ambiental y social del oleoturismo.

Al respecto, Northcote y Alonso (2011) destacan la ventaja competitiva de la que disfruta el oleoturismo, siempre y cuando se localice cerca de destinos turísticos consolidados, siendo fundamental la cooperación efectiva entre las empresas (públicas, privadas y/o mixtas), los servicios turísticos y los demás stakeholders existentes en el destino oleoturístico.

Los estudios de oleoturismo llevados a cabo hasta el momento, se han realizado desde la perspectiva de la oferta (Millán, 
Agudo y Morales, 2011; Millán y Morales, 2012; Duarte y Krajsic, 2013; Millán, Morales y Pérez, 2014; Moral, et al., 2014; Elías y Barbero, 2017); de la demanda (Molina, et al., 2011; Ruiz, et al., 2011; Cañero, et al., 2015; López-Guzmán, et al., 2016; Orgaz, et al., 2017; Millán, Pablo-Romero y SánchezRivas, 2018); o de ambos (Millán y Agudo, 2010; Millán, Morales y Agudo, 2010; Millán, Morales y Pérez-Naranjo, 2010; Millán, et al., 2011).

Orgaz-Agüera, et al. (2018), destacan la complejidad del oleoturismo y que implica a multitud de actores con diferentes intereses. Por ello, consideran fundamental conocer las percepciones, opiniones y actitudes de todos ellos para desarrollar turísticamente y de forma adecuada un destino, destacando el estudio de los stakeholders y sus interrelaciones y cooperación, como una línea de investigación poco estudiada y muy importante para la gestión del oleoturismo.

De este modo, este trabajo de investigación trata de caracterizar a las empresas pioneras en el desarrollo del oleoturismo y de conocer su compromiso, voluntad y realidad en materia de colaboración y cooperación con el resto de agentes del mercado oleoturístico. Se parte de la hipótesis de que las empresas oleoturísticas aún tienen un largo camino por recorrer en materia de colaboración entre actores, puesto que no tienen asumidos plenamente los beneficios de esta integración, relegándola a un segundo lugar en sus prioridades y focalizándola en relacionarse con la administración pública y aquellos operadores que les hagan vender más, dejando de lado a otros productores de aceite de oliva y a la población local.

\section{La colaboración entre actores en turismo, turismo rural, agroturismo y oleoturismo}

El vínculo existente entre turismo $\mathrm{y}$ sostenibilidad es muy fuerte. Un desarrollo sostenible del turismo es fundamental para el aprovechamiento de los efectos positivos del mismo, superando y minimizando los impactos negativos que pueda conllevar, permitiendo lograr un desarrollo sostenible en sus tres dimensiones: Social, económica y ambiental.

Bien es sabida la atención prestada en materia de sostenibilidad por parte de instituciones de carácter internacional como la UNESCO o la Organización Mundial del Turismo (Tregua, et al., 2018). Pero, además, como a continuación se refleja, la literatura científica muestra un gran interés en la identificación y el análisis de las relaciones que se establecen entre los diversos actores que afectan y se ven afectados por el turismo, puesto que es esencial para un desarrollo sostenible del turismo.

De este modo, el turismo debe analizarse como un sistema integrado entre los turistas y el territorio en el que se produce un intercambio constante entre las diferentes partes que lo componen (Merinero y Pulido, 2009), al tratarse de una actividad interdependiente e interrelacionada, en la que se amplifica el grado de exigencia sobre todos los agentes implicados en el desarrollo turístico, los cuales son múltiples y diversos.

La colaboración entre actores proporciona un proceso flexible y dinámico que evoluciona a lo largo del tiempo, lo que permite a las distintas partes interesadas abordar problemas de manera conjunta (Gray, 1989). De este modo, las organizaciones implicadas aúnan sus conocimientos, comparten experiencias, capital y otros recursos (Plummer, Telfer y Hashimoto, 2006), alcanzando la armonía y el consenso necesarios para aprovechar las oportunidades que surjan y lograr soluciones innovadoras, teniendo en cuenta los diferentes puntos de vista de las partes interesadas en el destino e identificando las distintas funciones y responsabilidades de cada una para que puedan contribuir al objetivo general de logar un desarrollo sostenible del turismo (Graci, 2013).

Este proceso de colaboración entre los stakeholders tiene su origen en la "Teoría de las partes interesadas" de Freeman (1984), con un enfoque desde la gestión empresarial, para 
ser adoptada por Murphy (1983) en materia de turismo, al establecer que para que la industria y el destino se vean beneficiados, es necesario instaurar relaciones simbióticas entre los actores.

El turismo, es una actividad en la que la colaboración entre actores es esencial para asegurar su desarrollo sostenible, y los participantes, que tradicionalmente han trabajado de un modo aislado, deben aprender y esforzarse en cooperar con el resto de las partes interesadas (Graci, 2013).

Esta colaboración entre actores puede realizarse de manera formal y estructurada, como los denominados acuerdos de administración conjunta o, sin embargo, los acuerdos de colaboración se pueden llevar a cabo a través de arreglos informales y mediante formas no estructuradas como las redes e iniciativas de base, que surgen temporalmente para resolver problemas específicos en el dominio (Jamal y Stronza, 2009).

En este sentido, Brown (1991) describió cuatro formas de colaboración entre actores, que van desde redes poco estructuradas hasta asociaciones $o$ coaliciones formalmente estructuradas: (1) Una red interorganizacional, (2) una asociación de organizaciones y redes, (3) una asociación intersectorial y (4) un movimiento social y coalición relacionada. Siendo necesario que las iniciativas de colaboración evolucionen a mayores niveles de organización, especialmente en dominios complejos de sostenibilidad, como es el caso de los destinos turísticos.

En el caso del turismo rural, donde se enmarca el oleoturismo, la relación de este con la sostenibilidad recibe una gran atención por parte de la literatura científica (Lane, 1994; Butler, 1999; Hardy, Beeton y Pearson, 2002; Hall, 2004; Garrod, Wornell y Youell, 2006; Blancas, et al., 2011, entre otros). La sostenibilidad de los recursos naturales y culturales, ya no puede gestionarse de un modo aislado y con partes interesadas independientes. A menudo, los impactos están interrelacionados $\mathrm{y}$, para abordar los problemas, es necesaria la participación de las partes interesadas (Jamal y Stronza, 2009).
En el turismo rural intervienen multitud de agentes, como el sector privado de distintos sectores de actividad que son productores de diferentes bienes y servicios turísticos; sector público (local, regional y nacional); agencias de viajes; organizaciones sin ánimo de lucro; la población local; operadores turísticos; centros de investigación; los turistas; entre otros. Los cuales deben estar integrados, y la ausencia de un marco integrador supone una fuerte debilidad (Bramwell y Sharman, 1999; Crouch y Ritchie, 1999; Mitchell y Eagles, 2001; Cawley y Gillmor, 2008).

De hecho, el establecimiento de redes entre actores y la colaboración entre estos, son considerados fundamentales para el éxito del turismo sostenible (McComb, Boyd y Boluk, 2017) y como parte de las bases del concepto desarrollado por la literatura científica de turismo rural integrado, cuyo objetivo es "promover la sostenibilidad ambiental, económica y sociocultural en el turismo y empoderar a la población local y contribuir así a la sostenibilidad del sistema rural más amplio" (Cawley y Gillmor, 2008, p.318).

Los primeros estudios en este sentido (Keogh, 1990; Lankford y Howard, 1994; Wearing y McDonald, 2002), destacaron que la colaboración entre actores puede ser muy beneficiosa para los destinos, puesto que implica la participación activa de los interesados en el desarrollo del turismo y porque se trata de una forma de gestión que favorece al mismo. Además, el capital social, entendido como el conjunto de relaciones que se dan entre las empresas, se ha de concebir como un proceso efectivo de intercambio de información y recursos que solo puede generarse entre personas (Anderson y Jack, 2010).

Sin embargo, es fundamental asumir que desarrollar la colaboración entre actores "per se", sin que el destino cumpla con unas condiciones mínima para su éxito (ver Tabla 1), puede suponer unos efectos devastadores (McComb, et al., 2017). De hecho, Spyriadis (2002) señala cómo más de la mitad de las relaciones de colaboración analizadas en estudios empíricos han resultado un fracaso. 


\section{Tabla 1}

\section{Condiciones para una colaboración efectiva entre partes interesadas}

\begin{tabular}{l} 
Condiciones \\
\hline 1. Correcta identificación de stakeholders. \\
2. Educación y capacitación para aquellos que carecen de conocimientos y habilidades para participar en el proceso. \\
3. Que las partes interesadas se involucren en el proceso. \\
4. Actividades de colaboración justas, eficientes, informadas, basadas en el conocimiento y estables. \\
5. Recursos suficientes. \\
6. Convocatoria legítima, con experiencia y conocimiento. \\
7. Las actividades utilizadas permiten la participación activa de los interesados. \\
8. Las partes interesadas creen que su participación tiene el potencial de influir en las decisiones. \\
9. Amplia gama de actividades utilizadas para involucrar a los interesados. \\
\hline
\end{tabular}

Fuente: McComb, et al. (2017).

El turismo rural y el agroturismo, están estrechamente relacionados y basados en recursos endógenos y de la propiedad, existiendo una gran vulnerabilidad de las empresas rurales a la competencia de la inversión externa, puesto que, cuanto mayor es la popularidad del destino, surgen conflictos entre el turismo, los recursos y los residentes (Cawley y Gillmor, 2008).

$\mathrm{La}$ colaboración entre empresas agrícolas contribuye al desarrollo sostenible del destino, puesto que además de los beneficios económicos, favorece la preservación de los recursos naturales, la creación de empleo y la protección de las tradiciones (Lebe y Milfelner, 2006; Dias y Franco, 2018).

En lo que al agroturismo se refiere, dado el papel destacado del sector privado para la implementación de estas actividades (Anuar, et al., 2012), la literatura científica destaca, que la cooperación entre los empresarios de las explotaciones agrícolas es una condición fundamental para la integración del turismo como una actividad complementaria que diversifique sus ingresos (Wilson, et al., 2001), de manera que se lleve a cabo desde una perspectiva de armonía dinámica entre las partes, siendo necesaria la integración vertical, horizontal y territorial de éstas.

Despotović, et al. (2017), realizan un estudio en explotaciones agrícolas y llegan a concluir que es necesario establecer clústeres en agroturismo para satisfacer los intereses conjuntos y promover las actividades de turismo rural. De este modo, al aunar esfuerzos se contribuye a la mejora de la calidad de los servicios prestados. La asociación mediante clúster:

Resulta de la vinculación de la agricultura con la artesanía y otras actividades, la producción especializada, la sensibilización sobre la importancia del agroturismo para las comunidades rurales, la oferta de nuevos servicios en las explotaciones y una mejor infraestructura. Así, las explotaciones se hacen más atractivas. (Despotović, et al., 2017, p.59)

En materia de oleoturismo, apostar por el desarrollo sostenible del turismo por parte de las empresas oleoturísticas, es fundamental, fomentando la potencialización del aceite de oliva, ante la cantidad de efectos positivos que supone (empleo, conservación de los recursos naturales y culturales), dinamizando la economía de la zona (Orgaz-Agüera, et al., 2018). A la hora de analizar la colaboración entre actores en materia de oleoturismo, la literatura científica es escasa.

La generación de ingresos de forma planificada en oleoturismo, requiere la coordinación e implicación de todos los 
agentes que intervienen en el proceso y deben tener en cuenta un entorno de calidad, que es la única garantía para ofrecer un producto turístico fruto del esfuerzo y la tenacidad de la población así como de los recursos de las áreas rurales (Millán, et al., 2010). La delimitación y desarrollo adecuados de acuerdos de colaboración, permiten un mejor conocimiento de la demanda potencial oleoturística y de las formas de estimular su interés.

En ese sentido, Millán, et al. (2014) llegan a marcar un planteamiento estratégico que debe colocar en práctica la comunidad del oleoturismo para convertir el aceite de oliva en un producto turístico referencia para la demanda turística. La coordinación, colaboración e integración entre actores, se refleja en cuatro de las seis estrategias que marca:

1. Coordinación de instituciones públicas y privadas, con la finalidad de promover el turismo culinario en general y del aceite de oliva en particular.

2. Integración de los diferentes negocios, de los distintos sectores, que participan del oleoturismo con el objetivo de diseñar una oferta integrada, que mitigue la estacionalidad de la demanda, impulsar la promoción turística conjunta y la participación asociada en determinados eventos.

3. Colaboración entre empresas e instituciones educativas, a fin de coordinar la formación reglada para satisfacer las necesidades de empresas e instituciones del sector.

4. Promover una cultura de cooperación entre los habitantes del destino, con el fin de facilitar la competencia leal, erradicar las actividades ilegales y mejorar así las perspectivas sociales del desarrollo turístico y que se conviertan en proveedores de información clave (Tregua, et al., 2018).

Como se ha expuesto hasta aquí, es palpable la importancia que la colaboración entre actores ha cobrado durante los últimos años en el desarrollo del oleoturismo. De igual forma, el desarrollo local de los entornos rurales necesita de esta cooperación (Quispe, Ayaviri y Maldonado, 2018). Sin embargo, aunque el cuerpo de literatura actual cuenta con diversas publicaciones que se han centrado en el desarrollo rural, la oferta oleoturística o la importancia que cobran los actores para la mejora de la comunidad local, no se ha cuestionado en profundidad cuál es su predisposición real a colaborar entre ellos, diferenciando los distintos stakeholders partícipes en la actividad turística.

\section{Metodología}

El presente estudio de tipo descriptivo, se ha llevado a cabo en empresas situadas en Andalucía cuya actividad económica está basada principalmente en el sector oleícola y/o prestan servicios relacionados con el aceite de oliva y su cultura. Los territorios en los que se ubican, se caracterizan por una fuerte dependencia de la agricultura, en este caso del cultivo del olivar (Hernández, Folgado y Campón, 2016).

Aunque la totalidad de las empresas encuestadas presentan, por los años de experiencia en el sector, una gran estabilidad, han decidido diversificar su línea de negocio hacia el turismo, siendo éste el hecho por el cual han sido seleccionadas para la realización de esta entrevista. A través de diversos metabuscadores, se identificaron aquellas empresas (ver Tabla 2) con mejor posicionamiento en la red y que, por tanto, mostrasen síntomas de haber avanzado en cuanto a la puesta en marcha de una propuesta oleoturística enfocada a la atracción de visitantes y turistas. Además, era condición sine qua non que tuviesen algún canal de reservas abierto a potenciales visitantes. Tras la búsqueda se identifican 38 empresas a las que se le envió la encuesta, de las que se obtuvieron 33 respuestas. 


\section{Tabla 2}

\section{Ficha técnica de la encuesta}

\begin{tabular}{|c|c|}
\hline Elemento & Observación \\
\hline Población & $\begin{array}{l}\text { Empresas que han diversificado su actividad hacia el turismo, en este caso más concreto, el } \\
\text { oleoturismo }\end{array}$ \\
\hline Ámbito & Andalucía \\
\hline Tipo de encuesta & $\begin{array}{l}\text { Cuestionario estructurado enviado por correo electrónico y contacto telefónico con los } \\
\text { gerentes y/o responsables de oleoturismo }\end{array}$ \\
\hline $\begin{array}{l}\text { Tamaño de la } \\
\text { muestra }\end{array}$ & 33 encuestas válidas \\
\hline Nivel de confianza & $95 \%$ \\
\hline Error muestral & $6,27 \%$ \\
\hline
\end{tabular}

Fuente: Elaboración propia, 2021.

Con el fin de obtener el mayor volumen de información posible acerca del desarrollo del oleoturismo en las empresas seleccionadas, que son pioneras en el desarrollo de esta tipología turística, se diseñó una encuesta que contiene cien preguntas que permiten caracterizar tanto a dichas empresas como a la actividad oleoturística que están llevando a cabo actualmente.

La encuesta, dividida en seis grandes bloques y un apartado final, está compuesta por preguntas dicotómicas y múltiples, cuya respuesta deriva a la exclusión o no de responder otras cuestiones y/o bloques. Respecto a las preguntas destinadas a medir variables, se ha empleado la escala de medición tipo Likert, con una puntuación de 1 a 7 , otorgando siempre la posibilidad al encuestado de añadir algún elemento adicional, si éste no ha sido contemplado.

Los resultados obtenidos de esta encuesta, han dado lugar a un amplio estudio de caracterización de la oferta y la demanda. En este artículo, en concreto, van a analizarse los resultados obtenidos con respecto a las relaciones y colaboración entre actores, que se centran en el objeto de estudio de esta investigación. Al tratarse de variables descriptivas, los resultados obtenidos se mostrarán en fracciones, medias y desviaciones típicas los datos obtenidos.

\section{Resultados y discusión}

En primer lugar, se trató de conocer con detalle la actividad empresarial que realizan las empresas mejor posicionadas en materia de oleoturismo, tal como se observa en la Tabla 3. De las treinta y tres encuestas realizadas a empresas oleoturísticas, la mayor parte de las respuestas provienen de almazaras, por lo que el $84,8 \%$ se dedica a la producción y venta de aceite de oliva y el resto se divide en museos de interpretación $(6 \%)$, servicios de guía $(3 \%)$ y otros $(6 \%)$. En definitiva, son tipos de empresas necesarias para el desarrollo del oleoturismo en un territorio (Cehic, Mesic y Oplanić, 2020). 
Tabla 3

Principal actividad de las empresas encuestadas

\begin{tabular}{lll}
\hline Principal actividad de las empresas encuestadas & No. & $\mathbf{\%}$ \\
\hline Alojamiento & 0 & 0 \\
Restauración & 0 & 0 \\
Agencia de Viajes & 0 & 0 \\
Servicios de guía & 1 & 3,03 \\
Producción y venta de aceite de oliva & 28 & 84,85 \\
Spa & 0 & \\
Museo centro de interpretación & 2 & 6,06 \\
Otro & 2 & 6,06 \\
Total & $\mathbf{3 3}$ & $\mathbf{1 0 0}$ \\
\hline
\end{tabular}

Fuente: Elaboración propia, 2021.

A su vez, profundizando con más detalle en la actividad principal de la empresa, el 57\% son empresas productoras, el $24 \%$ se dedican al envasado del aceite de oliva y el $3 \%$ a su comercialización (ver Tabla 4). No participó ninguna empresa que se dedique de un modo exclusivo a las actividades de laboratorio o de almacenamiento.

\section{Tabla 4}

\section{Detalle de la principal actividad de su empresa}

\begin{tabular}{lcc}
\hline \multicolumn{1}{c}{ Tipo de actividad } & No. & \% \\
\hline Productor & 19 & 57,58 \\
Envasador & 8 & 24,24 \\
Laboratorio & 0 & 0 \\
Almacenador & 0 & 0 \\
Comercializador & 1 & 3,03 \\
Otro & 5 & 15,15 \\
Total & $\mathbf{3 3}$ & $\mathbf{1 0 0}$ \\
\hline
\end{tabular}

Fuente: Elaboración propia, 2021.

Es fundamental conocer cuáles son los objetivos prioritarios de las empresas productoras y vendedoras (28), que son las que cuentan con más recursos e instalaciones para desarrollar productos turísticos en torno al oleoturismo y son las propietarias de marcas de aceite, para identificar qué motiva y estimula la actividad de estas empresas.

Los resultados son coincidentes (ver Tabla 5), la motivación principal de los encuestados gira en torno a acrecentar la cuota de mercado, de manera que la mayoría busca, en primer lugar, aumentar el prestigio de las marcas oleícolas que comercializan para atraer nuevos clientes $(64,28 \%)$, como ya indicaban Duarte y Northcote (2010), con respecto al oleoturismo como una actividad que permite mostrar sus productos a los visitantes. En segundo lugar, diversificar su oferta hacia el ámbito turístico $(53,57 \%)$, aunque el 21,4\% la señalan como primera prioridad $y$, por último, generar prescriptores de marcas oleícolas $(53,57 \%)$.

Tabla 5

Prioridad que otorga la empresa a los siguientes objetivos en torno al oleoturismo

\begin{tabular}{cccc}
\hline & $\begin{array}{c}\text { Aumentar el prestigio de } \\
\text { las marcas oleícolas que } \\
\text { comercializa para atraer } \\
\text { nuevos clientes }\end{array}$ & $\begin{array}{c}\text { Diversificar su } \\
\text { oferta hacia el } \\
\text { ámbito turístico }\end{array}$ & $\begin{array}{c}\text { Generar } \\
\text { prescriptores de } \\
\text { marcas oleícolas }\end{array}$ \\
\hline $1^{\text {a } \text { prioridad }}$ & 18 & 6 & 4 \\
$2^{\text {a } \text { prioridad }}$ & 4 & 15 & 9 \\
$3^{\text {a } \text { prioridad }}$ & 6 & 7 & 15 \\
\hline
\end{tabular}

Fuente: Elaboración propia, 2021. 
Además de estos objetivos, las empresas plantean otros en torno al oleoturismo, como son los que a continuación se detallan: 1. Promocionar su marca; 2. Formar al consumidor en el conocimiento de la elaboración del AOVE, contacto directo; 3. Incrementar la riqueza del entorno; 4. Vender más aceite; e. Fomentar la educación y la información; 5. Búsqueda de nuevos canales de venta e internacionalización de la empresa, con fórmulas diferentes de venta y promoción; 6. Dar a conocer la cultura y patrimonio; 7. Educar al consumidor en el aceite; 8. Visitar el pueblo; 9. Mejorar las instalaciones; 10. Dar prestigio a la empresa; 11. Alcanzar mayor difusión; y, 12. Situar el oleoturismo como actividad cultural, siendo destino de visitas culturales.
A la hora de comercializar el aceite de oliva, la empresa productora puede hacerlo de un modo directo o estableciendo canales y redes de distribución, que favorezcan la colaboración y la interacción entre actores. La Tabla 6, muestra los resultados de las empresas productoras y vendedoras en este sentido, a quienes se les preguntó cómo comercializan el aceite de oliva principalmente (realidad) y cómo desearían que se comercializara (expectativa). Puede observarse que la principal vía de comercialización, es mediante la venta directa y desean potenciar la misma a través de canales online, y reducir la que realizan a través de la gran distribución y agentes distribuidores.

Tabla 6

Formas principales de comercialización del aceite de oliva. Realidad y expectativa. $\mathrm{N}=\mathbf{2 8}$

\begin{tabular}{lcc}
\multicolumn{1}{c}{ Formas de comercialización } & Realidad & Expectativa \\
\hline A través de agentes distribuidores & 15 & 11 \\
A través de la gran distribución & 15 & 9 \\
Venta directa en almazara & 22 & 16 \\
Canales Online & 15 & 16 \\
Otro & 1 & 1 \\
\hline
\end{tabular}

Fuente: Elaboración propia, 2021.

A continuación, se describen los resultados del bloque de respuestas sobre voluntad y compromiso de los encuestados, puesto que este aspecto es fundamental para comprender su implicación en la industria turística y su voluntad de colaboración con diferentes actores.

En ese sentido, la Tabla 7 muestra que los encuestados están bastante dispuestos a aplicar principios de sostenibilidad en la gestión de su oferta oleoturística, como ya resaltaban Tregua, et al. (2018), así como a aplicar estándares de calidad a estos procesos para el desarrollo y gestión de sus propuestas oleoturísticas. La colaboración con otros actores, siendo competidores, queda relegada a un cuarto lugar, aunque muestran predisposición a ello. De hecho, el $100 \%$ de los encuestados estarían dispuestos como empresa a formar parte de una red de investigación, promoción y comercialización del oleoturismo. 


\section{Tabla 7}

\section{En base al éxito de su propuesta de oleoturismo, en qué medida estaría la} empresa dispuesta a... (Likert 1-7)

\begin{tabular}{lcc}
\hline $\mathbf{N}=\mathbf{3 3}$ & $\overline{\mathbf{x}}$ & $\boldsymbol{\sigma}$ \\
\hline $\begin{array}{l}\text {..Generar y compartir información estadística con un carácter periódico, bajo } \\
\text { una absoluta confidencialidad }\end{array}$ & 4,64 & 1,962 \\
...Adoptar ciertos procesos característicos de la industria turística & 5,21 & 1,431 \\
..Aplicar estándares de calidad a estos procesos para el desarrollo y gestión de \\
sus propuestas oleoturísticas \\
$\begin{array}{l}\text {...Colaborar con ciertos actores que, en principio, podría considerar } \\
\text { competidores }\end{array}$ \\
\begin{tabular}{l}
...Aplicar principios de sostenibilidad en la gestión de su oferta oleoturística \\
\hline
\end{tabular}
\end{tabular}

Fuente: Elaboración propia, 2021.

En relación con la creación de una red, y para comprobar su grado de interés e implicación, se les preguntó por la posibilidad de que su empresa realizara una inversión periódica con el objetivo de alcanzar sus fines oleoturísticos a través del desarrollo de esta red. Sin embargo, las respuestas no fueron tan unánimes, aceptando esta posibilidad el 60,6\% de los encuestados (20) y rechazándola el otro $39,4 \%(13)$.

En términos monetarios, la Tabla 8 muestra la cantidad que estarían dispuestas a invertir las empresas al año en dicha red de investigación, promoción y comercialización del oleoturismo. Puede observarse que la mayoría prefiere invertir la mínima cantidad posible.

\section{Tabla 8}

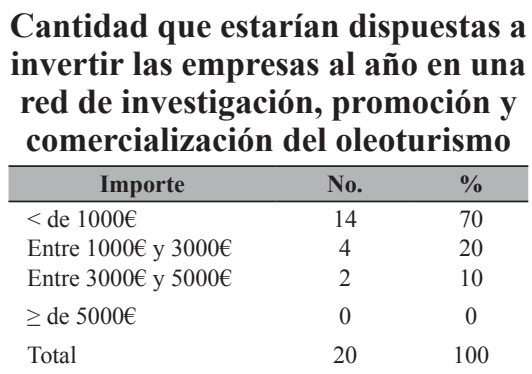

Fuente: Elaboración propia, 2021.
A continuación, se les preguntó, ya no por la disposición a establecer una red organizada, sino simplemente por su predisposición a colaborar con diferentes agentes para el desarrollo y éxito de su oferta oleoturística. Los resultados se reflejan en la Tabla 9. Las empresas encuestadas muestran mayor disposición para colaborar con la administración pública y otros productores turísticos del entorno, que no sean considerados competencia. Sin embargo, la posibilidad de cooperar con otros productores oleícolas es la que menor interés despierta, al identificarlas como competidores directos, aunque con elevado grado de disposición, al mostrar una valoración media próxima a 5 . 


\section{Tabla 9}

Disposición de las empresas a colaborar con los siguientes actores para el desarrollo y éxito de su oferta oleoturística. $\mathrm{N}=33$

\begin{tabular}{lcc}
\hline \multicolumn{1}{c}{ Actores } & $\overline{\mathrm{x}}$ & $\boldsymbol{\sigma}$ \\
\hline Otros productores oleícolas & 4,88 & 1,781 \\
Otros productores turísticos del entorno (restaurantes, hoteles, transporte...) & 6 & 1,199 \\
Empresas de otros sectores & 5,15 & 1,642 \\
Operadores turísticos & 5,91 & 1,422 \\
Agencias de viaje & 5,97 & 1,468 \\
Centros universitarios y de investigación & 5,91 & 1,234 \\
Administración pública (ayuntamientos, Diputación, comunidad autónoma...) & 6,09 & 0,914 \\
Población local & 5,63 & 1,220 \\
\hline
\end{tabular}

Fuente: Elaboración propia, 2021.

Al centrar las preguntas en el mercado oleoturístico, de nuevo, ningún actor del mercado oleoturístico recibe valoraciones inferiores a 4,5 al evaluar la importancia de la colaboración con estos, lo que evidencia, en cierta medida, la concienciación de las empresas oleoturísticas sobre la importancia de colaborar con el resto de actores para el éxito de su oferta (Arikan, 2017; Arıkan y Çeken, 2017)
Los encuestados consideran fundamental la colaboración con otras empresas turísticas (que no sean empresas oleoturísticas, ni oleícolas, que quedan relegadas a las últimas posiciones) y con los turistas. Las universidades y centros de investigación, son consideradas como aliadas en su desarrollo oleoturístico, dándoles la tercera posición según su valoración (ver Tabla 10).

Tabla 10

\begin{tabular}{|c|c|c|}
\hline Actores & $\bar{x}$ & $\sigma$ \\
\hline Otras empresas oleoturísticas & 5,15 & 1,716 \\
\hline Otras empresas oleícolas & 4,58 & 1,937 \\
\hline Otras empresas turísticas & 5,64 & 1,388 \\
\hline Empresas de otros sectores & 4,91 & 1,646 \\
\hline Población local & 4,97 & 1,630 \\
\hline Universidades y centros de investigación & 5,27 & 1,625 \\
\hline Turistas & 5,67 & 1,384 \\
\hline
\end{tabular}

Fuente: Elaboración propia, 2021.

Sin embargo, con respecto a otros oferentes de oleoturismo (ver Tabla 11), las empresas encuestadas tienen claro que debería existir una mayor colaboración en proyectos conjuntos $(81,81 \%)$ y que son colaboradores necesarios para competir en un mundo globalizado, descartando que sean competidores y nunca se pondrán de acuerdo $(3,03 \%)$. 


\section{Tabla 11}

Con respecto a otros oferentes de oleoturismo, las empresas consideran que... (Respuesta múltiple)

\begin{tabular}{lc}
\hline & No. \\
\hline Debería existir una mayor colaboración en proyectos conjuntos & 27 \\
\hline Ellos no tienen ni idea y por eso fracasarán, antes o después & 1 \\
\hline Ofrecen algo excepcional y mi empresa debe copiar la idea & 1 \\
\hline $\begin{array}{l}\text { Ellos llevan más tiempo que mi organización ofreciendo oleoturismo, pero mi empresa ofrece algo mejor } \\
\text { en algunos aspectos }\end{array}$ & 5 \\
\hline Ellos poseen ventajas competitivas importantes & 4 \\
\hline Ellos son colaboradores necesarios para competir en un mundo globalizado & 11 \\
\hline Ellos son competidores y nunca nos pondremos de acuerdo & 1 \\
\hline Otro & 0 \\
\hline
\end{tabular}

Fuente: Elaboración propia, 2021.

A la hora de establecer los roles de cada actor en materia de colaboración para la creación del producto oleoturístico, los encuestados, en su mayoría, piensan que ellos mismos $(75,8 \%)$, junto con las administraciones públicas (Diputación en un $75,8 \%$, Ayuntamientos en un $66,67 \%$ y
Junta de Andalucía con un 66,6\%) deberían asumir el liderazgo del proceso. Otorgan, sin embargo, un papel secundario, aunque con menor consenso, a otros oferentes: Los turistas, los centros de estudio e investigación y la población local (ver Tabla 12).

Tabla 12

Actitud que debe adoptar cada uno de los siguientes actores en cuanto a la creación del producto oleoturístico

\begin{tabular}{lccc}
\hline \multicolumn{1}{c}{ Actores } & $\begin{array}{c}\text { Debe asumir el } \\
\text { liderazgo del } \\
\text { proceso }\end{array}$ & $\begin{array}{c}\text { No debe } \\
\text { liderar, sino } \\
\text { seguir a quien } \\
\text { lidera el } \\
\text { proceso }\end{array}$ & $\begin{array}{c}\text { Debe } \\
\text { permanecer } \\
\text { indiferente/ } \\
\text { pasivo }\end{array}$ \\
\hline Su empresa & 25 & 8 & 0 \\
Otros oferentes de oleoturismo & 15 & 16 & 2 \\
Diputación Provincial & 25 & 6 & 2 \\
Operadores turísticos & 17 & 14 & 2 \\
Junta de Andalucía & 20 & 13 & 0 \\
Turistas & 15 & 17 & 1 \\
$\begin{array}{l}\text { Ayuntamiento donde se encuentra su oferta } \\
\text { oleoturística }\end{array}$ & 22 & 10 & 1 \\
$\begin{array}{l}\text { Otros organismos (Asociación Española } \\
\text { de Municipios del Olivo, Consejo Oleícola }\end{array}$ & 17 & 15 & 1 \\
Internacional...) & & 17 & 1 \\
Centros de estudio e investigación & 15 & 17 & 5 \\
Población local & 11 & 17 & \\
\hline
\end{tabular}

Fuente: Elaboración propia, 2021. 
Respecto a la actitud que consideran los encuestados que deben adoptar los distintos actores en materia de colaboración para la promoción y comercialización del producto oleoturístico, sí que piensan que el liderazgo debe ser asumido de un modo conjunto entre su propia empresa $(81,8 \%)$, otros oferentes del oleoturismo $(66,7 \%)$, el sector público (Diputación en un 78,8\%, Ayuntamientos en un $66,7 \%$ y Junta de Andalucía con un $75,7 \%$ ) y los operadores turísticos $(69,7 \%)$ (ver Tabla 13).

Tabla 13

Actitud que consideran las empresas que debe adoptar cada uno de los siguientes actores en cuanto a la promoción y comercialización del producto oleoturístico

\begin{tabular}{lccc}
\hline \multicolumn{1}{c}{ Actores } & $\begin{array}{c}\text { Debe asumir el } \\
\text { liderazgo del } \\
\text { proceso }\end{array}$ & $\begin{array}{c}\text { No debe liderar, } \\
\text { sino seguir a quien } \\
\text { lidera el proceso }\end{array}$ & $\begin{array}{c}\text { Debe permanecer } \\
\text { indiferente/ } \\
\text { pasivo }\end{array}$ \\
\hline Su empresa & 27 & 6 & 0 \\
Otros oferentes de oleoturismo & 22 & 8 & 3 \\
Diputación Provincial & 26 & 4 & 3 \\
Operadores turísticos & 23 & 8 & 2 \\
Junta de Andalucía & 25 & 7 & 1 \\
Turistas & 13 & 18 & 2 \\
$\begin{array}{l}\text { Ayuntamiento donde se encuentra su } \\
\text { oferta oleoturística }\end{array}$ & 22 & 9 & 1 \\
Otros organismos (Asociación & 16 & 16 & 1 \\
Española de Municipios del Olivo...) & 15 & 16 & 2 \\
Centros de estudio e investigación & 9 & 21 & 3 \\
Población local & & & \\
\hline
\end{tabular}

Fuente: Elaboración propia, 2021.

Por último, se realizaron una serie de preguntas para conocer la realidad de la colaboración de las empresas estudiadas con otros actores. El $57,57 \%$ de los encuestados (19) mantienen acuerdos de colaboración en materia de oleoturismo con otras organizaciones, siendo ésta estable en un $75 \%$ de los casos (un $43 \%$ del total). Se trata de una colaboración que habitualmente inicia la propia empresa $(75 \%)$ y está basada, en un $65 \%$ de los casos, en acuerdos y convenios formales, siendo el resto de carácter verbal.

Asimismo, las empresas participantes, en una sección de respuestas libres para indicar otras cualidades no contempladas de la colaboración de su empresa con otras organizaciones en materia oleoturística, destacaron que: "En algunos aspectos cuesta trabajo conseguir convencer a otros de la necesidad de colaborar para desarrollar proyectos conjuntos beneficiosos para ambas partes, debido a la falta de políticas colaborativas".

\section{Conclusiones}

El oleoturismo, es una forma de turismo que se enmarca en el turismo rural, agroturismo, gastronómico, comunitario, e, incluso, dentro del turismo industrial, a través del cual se profundiza en la cultura del olivar y todo lo relacionado con el proceso de 
extracción, propiedades y empleo del aceite de oliva.

Así, la cooperación y colaboración entre actores es fundamental para abordar los problemas que surjan a lo largo del desarrollo turístico sostenible, a través de un proceso flexible y dinámico, de manera que las partes interesadas coloquen en común conocimientos, experiencia, capital así como recursos, y entre todas puedan enriquecerse de esa puesta en común.

En ese sentido, el presente estudio ha permitido caracterizar a las empresas pioneras en el desarrollo del oleoturismo, además de conocer su compromiso, voluntad y realidad en materia de colaboración y cooperación con el resto de partes interesadas así como agentes del mercado oleoturístico.

Las empresas encuestadas son en su mayoría productoras y comercializadoras de aceite de oliva, cuyos objetivos principales giran en torno a la meta de "vender más". La formación y participación en redes de colaboración y la cooperación con otros actores, no entra dentro de sus objetivos principales e inmediatos, aunque sí la tienen en alta consideración.

A la hora de comercializar el aceite de oliva, apuestan por la venta directa in situ u online. No prestan atención suficiente a la posibilidad de trabajar con agentes o redes de distribución de sus productos, que favorezcan la colaboración o el trabajo integrado inter o intrasectorial.

Respecto a la realidad en materia de relaciones estables de colaboración con otros agentes por parte de las empresas encuestadas, no se trata de una práctica extendida en la mayoría de éstas (43\%). Destacan la dificultad para convencer a las demás partes de los beneficios de esta integración.

Ciertamente, son los propios encuestados los que muestran disposición a la colaboración, pero con ciertos matices. En primer lugar, ésta no se encuentra entre sus prioridades más inmediatas y su predisposición para invertir económicamente en redes de colaboración, participación o comercialización del oleoturismo es mínima.
Además, para ellos, la colaboración con otros actores se centra, principalmente, en el sector público, otros productores turísticos (no competidores) y operadores, lo cual conlleva que deseen asumir el liderazgo de la colaboración entre actores de la mano del sector público. Finalmente, la población del destino apenas es considerada como un actor al que deben tener en cuenta.

A modo de resumen, puede decirse que, en materia de colaboración entre las partes interesadas del mercado oleoturístico, las empresas con mejor posicionamiento en esta actividad necesitan información, formación e incentivos, para desarrollar el oleoturismo a través de la participación entre actores, sin excluir ni relegar a ninguno: Productores de aceite de oliva, tejido empresarial del destino, sector público y la población.

Una sociedad rectora, como señalan Tregua, et al. (2018), es fundamental en este sentido, de manera que impulse a las empresas oleoturísticas a la colaboración sin prejuicios y con decisión así como ayude a superar las debilidades y amenazas con las que se encuentran, apoyándose en las fortalezas de cada una de ellas y las oportunidades del mercado.

\section{Referencias bibliográficas}

Alonso, A. M., y Guzmán, G. (25 de mayo de 1999). Cultivo del olivar en la agricultura ecológica. Hoja divulgativa 2/99 del Comité Andaluz de Agricultura Ecológica, Consejería de Agricultura y Pesca de la Junta de Andalucía. Boletinagrario. com. https://boletinagrario.com/ f134, cultivo-olivar-agriculturaecologica.html

Anderson, A. R., y Jack, S. L. (2010). The articulation of social capital in entrepreneurial networks: A glue or a lubricant? Entrepreneurship \& Regional Development: An International Journal, 
Carrillo-Hidalgo, Isabel; Casado-Montilla, Jairo y Pulido-Fernández, Juan Ignacio

Desarrollo sostenible del oleoturismo: Un análisis de la colaboración entre actores

14(3), $\quad 193-210 . \quad \underline{\text { https://doi. }}$
org/10.1080/08985620110112079

Anuar, A., Ahmed, H., Jusoh, H., y Hussain, M. (2012). Understanding the role of stakeholder in the formation of tourist friendly destination concept. Journal of Management and Sustainability, 2(1), 106-114. https://doi.org/10.5539/ ¡ms.v2n1p106

Arikan, I. (2017). Initiative activities of olive oil tourism: A case from turkey. Journal of Academic Social Sciences, 5(55), 134-151. http://dx.doi.org/10.16992/ ASOS.12790

Arikan, I., y Çeken, H. (2017). New trends in agrotourism: Olio tourism. Journal of Life Economics, 4(4), 89-102. https:// doi.org/10.15637/jlecon.212

Aybar, R. (2004). Proyecto Oleoturismo: Una red europea para la promoción de la cultura del olivo. Diputación Provincial de Jaén, España. http:// www.expoliva.com/expoliva $2005 /$ simposium/comunicaciones/CULT04.pdf

Blancas, F. J., Lozano-Oyola, M., González, M., Guerrero, F. M., y Caballero, R. (2011). How to use sustainability indicators for tourism planning: The case of rural tourism in Andalusia (Spain). Science of the Total Environment, 412413, 28-45. https://doi.org/10.1016/j. scitotenv.2011.09.066

Bramwell, B., y Sharman, A. (1999). Collaboration in local tourism policymaking. Annals of Tourism Research, 26(2), 392-415. https://doi. org/10.1016/S0160-7383(98)00105-4

Brown, L. D. (1991). Bridging organizations and sustainable development. Human Relations, 44(8), 807-831. https://bit. ly/32nCS51

Butler, R. W. (1999). Sustainable tourism: A state $\square$ of $\square$ the $\square$ art review. Tourism Geographies, 1(1), 7-25. https://doi. org/10.1080/14616689908721291

Campón-Cerro, A. M., Di-Clemente, E., Hernández-Mogollón, J. M., De Salvo, P., y Calzati, V. (2014). Olive oil tourism in southern Europe: Proposals for tourism development of olive grove rural areas. Turismo \& Desenvolvimento, (21/22), 63-73.

Cañero, P. M., López-Guzmán, T. J., Moral, S., y Orgaz, F. (2015). Análisis de la demanda del oleoturismo en Andalucía. Revista de Estudios Regionales, (104), 133-149.

Cawley, M., y Gillmor, D. A. (2008). Integrated rural tourism: Concepts and practice. Annals of Tourism Research, 35(2), 316-337. https://doi.org/10.1016/j. annals.2007.07.011

Cehic, A., Mesic, Ž., Oplanić, M. (2020). Requirements for development of olive tourism: The case of Croatia. Tourism Hospitality Management, 26(1), 1-14. https://doi.org/10.20867/ thm.26.1.1

Consejo Oleícola Internacional - COI (2018). Cifras aceites de oliva, noviembre 2018. COI. http://www. internationaloliveoil.org/estaticos/ view/131-world-olive-oil-figures

Crouch, G. I., y Ritchie, J. B. (1999). Tourism, competitiveness, and societal prosperity. Journal of Business Research, 44(3), 137-152. https://doi. org/10.1016/S0148-2963(97)00196-3

De Salvo, P., Hernández, J. M., Di Clemente, E., y Calzati, V. (2013). Territory, tourism and local products. The extra virgin oil's enhancement and promotion: a benchmarking Italy-Spain. Tourism and Hospitality Management, 19(1), 23-34.

Despotović, A., Joksimović, M., Svržnjak, K., y Jovanović, M. (2017). Rural areas sustainability: Agricultural Diversification and Opportunities 
for Agri-tourism Development. Agriculture and Forestry, 63(3), 47-62. $\quad$ https://doi.org/10.17707/ AgricultForest.63.3.06

Dias, C., y Franco, M. (2018). Cooperation in tradition or tradition in cooperation? Networks of agricultural entrepreneurs. Land Use Policy, 71, 36-48. https://doi. org/10.1016/j.landusepol.2017.11.041

Duarte, A. (2010). Olives, hospitality and tourism: A Western Australian perspective. British Food Journal, 112(1), 55-68. $\quad$ http://dx.doi. org/10.1108/00070701011011209

Duarte, A., y Krajsic, V. (2013). Food heritage down under: olive growers as Mediterranean 'food ambassadors. Journal of Heritage Tourism, 8(2-3), 158-171. https://doi.org/10.1080/1743 $\underline{873 X .2013 .767807}$

Duarte, A., y Northcote, J. (2010). The development of olive tourism in Western Australia: A case study of an emerging tourism industry. International Journal of Tourism Research, 12(6), 696-708. https://doi. org $/ 10.1002 / j$ tr. 786

Elías, S. R., y Barbero, A. C. (2017). Situación del oleoturismo y lineamientos para su desarrollo en la región del sudoeste bonaerense, Argentina. RIAT - Revista Interamericana de Medioambiente y Turismo 13(1), 91104. http://dx.doi.org/10.4067/S0718235X2017000100091

Freeman, R. E. (1984). Strategic management: A stakeholder approach. Pitman.

Garrod, B., Wornell, R., y Youell, R. (2006). Re-conceptualising rural resources as countryside capital: The case of rural tourism. Journal of Rural Studies, 22(1), 117-128. https://doi. org/10.1016/j.jrurstud.2005.08.001

Graci, S. (2013). Collaboration and partnership development for sustainable tourism.
Tourism Geographies, 15(1), 25-42. https://doi.org/10.1080/14616688.201 $\underline{2.675513}$

Gray, B. (1989). Collaborating: Finding common ground for multiparty problems. Jossey-Bass.

Hall, D. (2004). Rural tourism development in southeastern Europe: Transition and the search for sustainability. International Journal of Tourism Research, 6(3), 165-176. https://doi. org/10.1002/jtr.482

Hardy, A., Beeton, R. J. S., y Pearson, L. (2002). Sustainable tourism: An overview of the concept and its position in relation to conceptualisations of tourism. Journal of Sustainable Tourism, 10(6), 475-496. https://doi. org/10.1080/09669580208667183

Henderson, J. C. (2009). Food tourism reviewed. British Food Journal, 111(4), 317-326. https://doi. org/10.1108/00070700910951470

Hernández, J. M., Folgado, J. A., y Campón, A. M. (2016). Oleoturismo en la Sierra de Gata y las Hurdes (Cáceres): Análisis de su potencial a través de un test de producto. International Journal of Scientific Management and Tourism, 2(1), 333-354.

Jamal, T., y Stronza, A. (2009). Collaboration theory and tourism practice in protected areas: Stakeholders, structuring and sustainability. Journal of Sustainable Tourism, 17(2), 169-189. https://doi. org/10.1080/09669580802495741

Kizos, T., y Vakoufaris, H. (2011). Valorisation of a local asset: The case of olive oil on Lesvos Island, Greece. Food Policy, 36(5), 705-714. https://doi. org/10.1016/j.foodpol.2011.06.005

Keogh, B. (1990). Public participation in community tourism planning. Annals of Tourism Research, 17(3), 449465. https://doi.org/10.1016/0160- 
Carrillo-Hidalgo, Isabel; Casado-Montilla, Jairo y Pulido-Fernández, Juan Ignacio

Desarrollo sostenible del oleoturismo: Un análisis de la colaboración entre actores

\section{3(90)90009-G}

Lane, B. (1994). Sustainable rural tourism strategies: A tool for development and conservation. Journal of Sustainable Tourism, 2(1-2), 102-111. https://doi. org/10.1080/09669589409510687

Lankford, S. V., y Howard D. R. (1994). Developing a tourism impacts attitude scale. Annals of Tourism Research, 21(1), 121-139. https://doi. org/10.1016/0160-7383(94)90008-6

Lebe, S. S., y Milfelner B. (2006). Innovative organisation approach to sustainable tourism development in rural areas. Kybernetes 35(7/8), 1136-1146.https:// doi.org/10.1108/03684920610675139

Lee, K. H., y Scott, N. (2015). Food tourism reviewed using the paradigm funnel approach. Journal of Culinary Science \& Technology, 13, 95-115. https://doi. org/10.1080/15428052.2014.952480

Loor, L., Plaza, N., y Medina, Z. (2021). Turismo comunitario en Ecuador: Apuntes en tiempos de pandemia. Revista de Ciencias Sociales (Ve), XXVII(1), 265-277.

López-Guzmán, T., Cañero, P. M., Moral, S., y Orgaz-Agüera, F. (2016). An exploratory study of olive tourism consumers. Tourism and Hospitality Management, 22(1), 57-68. https://doi. org/10.20867/thm.22.1.1

McComb, E. J., Boyd, S., y Boluk, K. (2017). Stakeholder collaboration: A means to the success of rural tourism destinations? A critical evaluation of the existence of stakeholder collaboration within the Mournes, Northern Ireland. Tourism and Hospitality Research, 17(3), 286-297. https://bit.ly/3st038W

Merinero, R., y Pulido, J. I. (2009). Desarrollo turístico y dinámica relacional. Metodología de análisis para la gestión activa de destinos turísticos.
Cuadernos de Turismo, (23), 173-194.

Millán M. G., Agudo, E. M., y Agudo, G. I. (2010). Oil-tourism in the Southeast of Spain: The necessity of coordinating the tourist activity with the agrarian one for the development of the rural areas. Panorama Socioeconómico, 28(41), 116-124.

Millán, M. G., Agudo, E. M., y Morales, E. (2011). Análisis de la oferta y la demanda de oleoturismo en el sur de España: un estudio de caso. Cuadernos de Desarrollo Rural, 8(67), 22.

Millán, G., Arjona, J. M., y Amador, L. (2014). A new market segment for olive oil: Olive oil tourism in the south of Spain. Agricultural Sciences, 5(3), 179. http:// dx.doi.org/10.4236/as.2014.53022

Millán, M. G., Morales, E., y Agudo, E. M. (2010). El oleoturismo como motor de desarrollo rural: La denominación de origen de montoro-adamuz. Mundo Agrario, 11(21), 1-28.

Millán, M. G., Morales, E., y Pérez, L. M. (2014). Turismo gastronómico, Denominaciones de Origen y desarrollo rural en Andalucía: Situación actual. Boletín de la Asociación de Geógrafos Españoles, (65), 113-137. https://doi. org/10.21138/bage. 1746

Millán, M. G., Morales, E., y Pérez-Naranjo, L. M. (2010). Olive oil tourism as a vehicle for rural development in the province of Cordoba. Turismo \& Desenvolvimento, (13/14), 739-748.

Millán, M. G., Morales-Fernández, E. J., y Pérez, L. (2012). Análisis del turismo gastronómico en la provincia de Córdoba. Tourism \& Management Studies, (8), 78-87.

Millán, M. G., Pablo-Romero, M. D. P., y Sánchez-Rivas, J. (2018). Oleotourism as a sustainable product: An analysis of its demand in the south of Spain (Andalusia). Sustainability, 10(1), 101. 
https://doi.org/10.3390/su10010101

Millán, M. G., y Agudo, E. M. (2010). El turismo gastronómico y las Denominaciones de Origen en el sur de España: Oleoturismo. Un estudio de caso. Pasos - Revista de Turismo y Patrimonio Cultural, 8(1), 91-112. $\quad$ https://doi.org/10.25145/j. pasos.2010.08.008

Millán, M. G., y Morales, E. (2012). Denominaciones de Origen Protegidas (DOP) y turismo gastronómico: Una relación simbiótica en Andalucía. Gran Tour, (6), 101-121.

Mitchell, R. E., y Eagles, P. F. J. (2001). An integrative approach to tourism: Lessons from the Andes of Peru. Journal of Sustainable Tourism, 9(1), 4-28. https://doi. org/10.1080/09669580108667386

Molina, V., Quesada, J. M., y Ruiz, I. (2011). Potencial del oleoturismo como diversificación económica del sector cooperativo agrario: el caso español. Revista de Ciencias Sociales (Ve), XVII(3), 533-541.

Moral, S., Cañero, P. M., Orgaz, F., y LópezGuzmán, T. (2014). Una aproximación al oleoturismo en Andalucía, España. International Journal of World of Tourism, 1(2), 29-40. https://doi. org/10.12795/IJWT.2014.i02.04

Murgado, E. M. (2013). Turning food into a gastronomic experience: Olive oil tourism. Options Mediterranéennes, (106). 97-109.

Murphy, P. E. (1983). Tourism as a community industry. An ecological model of tourism development. Tourism Management, 4(3), 180193. https://doi.org/10.1016/02615177(83)

Northcote, J., y Alonso, A. D. (2011). Factors underlying farm diversification: the case of Western Australia's olive farmers. Agriculture and Human Values, 28(2), 237-246. https://doi. org/10.1007/s10460-010-9274-X

Ordoñez, A., y Ochoa, P. (2020). Ambiente, sociedad y turismo comunitario: La etnia Saraguro en Loja - Ecuador. Revista de Ciencias Sociales (Ve), XXVI(2), 180-191. https://doi. org/10.31876/rcs.v26i2.32433

Orgaz, F., Moral, S., López-Guzmán, T., y Cañero, P. (2017). Estudio de la demanda existente en torno al oleoturismo. El caso de Andalucía. Cuadernos de Turismo, (39), 437453. $\quad$ http://dx.doi.org/10.6018/ turismo.39.290641

Orgaz-Agüera, F., Moral, S., López-Guzmán, T., y Cañero, P. (2018). Características generales del oleoturismo y propuestas para futuras líneas de investigación. Estudios y Perspectivas en Turismo, 27(3), 487-505.

Plummer, R., Telfer, D., y Hashimoto, A. (2006). The rise and fall of the Waterloo-Wellington Ale Trail: A study of collaboration within the tourism industry. Current Issues in Tourism, 9(3), 191-205. https://doi. org/10.2167/cit/194.0

Pulido-Fernández, J. I., Casado-Montilla, J., y Carrillo-Hidalgo, I. (2019). Introducing olive-oil tourism as a special interest tourism. Heliyon, 5(12). $\quad$ https://doi.org/10.1016/j. heliyon.2019.e02975

Quesada, J. M., Molina, V., y Ruiz, I. (2010). Oleoturismo en España: Potencialidad de éxito internacional en escenarios actuales. Global Conference on Business and Finance Proceedings, 5(2), 1534-1560.

Quispe, G., Ayaviri, D., y Maldonado, R. (2018). Participación de los actores en el desarrollo local en entornos rurales. Revista de Ciencias Sociales (Ve), 
Carrillo-Hidalgo, Isabel; Casado-Montilla, Jairo y Pulido-Fernández, Juan Ignacio

Desarrollo sostenible del oleoturismo: Un análisis de la colaboración entre actores

$X X I V(3), 62-82$.

Ruiz, I. (2010). Análisis cuantitativo y cualitativo del significado del aceite de oliva. Una aproximación desde el punto de vista del consumidor (Tesis doctoral). Universidad de Granada, Granada, España.

Ruiz, I., Molina, V., y Martín, V. M. (2011). El oleoturismo como atractivo turístico en el medio rural español. Papers de Turisme, (49-50), 89-103.

Spyriadis A. (2002). Collaboration partnerships as strategic marketing tools of international hotel chains in pursuit of business development and competitive advantage in the global marketplace (MSc Dissertation). Bournemouth University, UK.

Tregua, M., D’Auria, A., y Marano-Marcolini, C. (2018). Oleotourism: Local actors for local tourism development. Sustainability, 10(5), 1492. https://doi. org/10.3390/su10051492

Wearing, S., y McDonald, M. (2002). The development of community-based tourism: Re-thinking the relationship between tour operators and development agents as intermediaries in rural and isolated area communities. Journal of Sustainable Tourism, 10(3), 191-206. https://doi. org/10.1080/09669580208667162

Wilson, S., Fesenmaier, D. R., Fesenmaier, J., y Van Es, J. C. (2001). Factors for success in rural tourism development. Journal of Travel Research, 40(2), 132-138. https://doi.org/10.1177\% $\underline{\text { 2F004728750104000203 }}$ 